01

\title{
Оптимизация уравнения для вычисления коэффициента заполнения при проектировании сборок Хальбаха
}

\author{
(C) F. Balcı ${ }^{1}$, A. Bingolbali ${ }^{1,}$, N. Dogan ${ }^{2}$, M. Irfan ${ }^{3}$ \\ ${ }^{1}$ Department of Bioengineering, Yıldız Technical University, Istanbul, Turkey \\ ${ }^{2}$ Department of Physics, Gebze Technical University, Gebze, Turkey \\ ${ }^{3}$ Department of Electronics Engineering, Gebze Technical University, Gebze, Turkey \\ "E-mail: ab1353@gmail.com
}

Поступило в Редакцию 12 мая 2020г.

В окончательной редакции 10 октября 2020 г.

Принято к публикации 18 октября 2020г.

В рамках задачи магнитопорошкового и магнитно-резонансного формирования изображений проведено моделирование поля выбора (пространственно неоднородного) и поля возбуждения (пространственно однородного), создаваемых с помощью магнитов Хальбаха. В области применения структур Хальбаха ключевыми факторами являются характеристики постоянных магнитов (длина и остаточная плотность магнитного потока) и геометрические параметры сборки (фактор заполнения $(F F)$, число магнитов и радиус структуры). Влияние отношения факторов заполнения на плотность магнитного потока исследовалось на схемах с четырьмя и восемью цилиндрическими магнитами. Выведено новое математическое выражение, позволяющее точно рассчитать необходимое расположение магнитов даже для случая $100 \%$ заполнения $(F F=1)$. Проведен численный расчет согласно предложенной модели, при этом точность определения плотности магнитного потока в четырехмагнитной системе на $17 \%$ превысила точность, обеспечиваемую моделью, предложенной в литературе.

Ключевые слова: магниты Хальбаха, фактор заполнения $(F F)$, магнитопорошковое формирование изображения (MPI), магнитно-резонансное формирование изображений (MRI).

DOI: 10.21883/PJTF.2021.04.50635.18453

Магнитная сборка Хальбаха представляет собой конфигурацию постоянных магнитов, обеспечивающую, с одной стороны, усиление магнитного потока, а с другой - его уменьшение вплоть до полного исчезновения. Такие двумерные схемы распределения намагниченности были впервые предложены Маллинсоном в 1971 г. [1]. Дальнейшая разработка таких схем распределения намагниченности была проведена Хальбахом [2,3], в результате чего были созданы системы многополярных постоянных магнитов, которые в течение последних десятилетий известны как сборки Хальбаха. Известны конфигурации магнитов Хальбаха линейного, кругового или сферического типа. Такие конфигурации магнитов могут создавать биполярные или многополярные магнитные поля.

Фактор заполнения [4] является одним из наиболее важных параметров магнитов Хальбаха, определяющим их эксплуатационные характеристики и применимость в различных областях (см. работы [5-15]), например в методах магнитопорошкового (MPI) и магнитнорезонансного (MRI) формирования изображений в медицинских целях. Фактор заполнения $(F F)$, упоминаемый при описании конфигурации магнитов Хальбаха, представляет собой коэффициент заполнения магнитов такой системы.

Значение $F F$ существенно влияет на изменение плотности магнитного потока. Целью настоящей работы является оптимизация приведенного в работе [4] уравнения для расчета фактора заполнения магнита Хальбаха, недостатком которого является отождествление диаметра магнита с длиной занимаемой им дуги. Кроме того, это уравнение подразумевает перекрытие расположенных следом друг за другом магнитов при $100 \%$ заполнении $(F F=1)$. Из-за этих особенностей используемое в литературе уравнение для определения $F F$ нельзя считать корректным.

В результате развития предложенной здесь математической модели удалось построить такую конфигурацию магнитов (сборку Хальбаха), при которой коэффициент заполнения, равный 1 , обеспечивается без взаимного перекрытия соседних магнитов. В работе исследуется влияние значения $F F$ на плотность магнитного потока в системах Хальбаха с четырьмя и восемью магнитами (М). Чтобы выделить влияние именно величины $F F$, значения всех остальных параметров (длины, остаточной магнитной индукции, радиуса структуры) поддерживались на постоянном уровне. Оптимизация всех параметров была также проведена, и влияние их значений на характеристики сборки Хальбаха было тщательно изучено [16]. На рис. 1 представлена геометрическая схема сборки Хальбаха с четырьмя магнитами.

Радиус магнита $|C P|$ выражается через центральный угол $(C O P)$, обозначенный как угол $\alpha$ :

$$
|C P|=\frac{D_{m}}{2}=R_{s} \sin (\alpha),
$$

где $\frac{D_{m}}{2}$ - радиус магнита, а $R_{s}$ - радиус сборки. Поскольку полный угол в точке $O$ составляет $2 \pi=360^{\circ}$, 


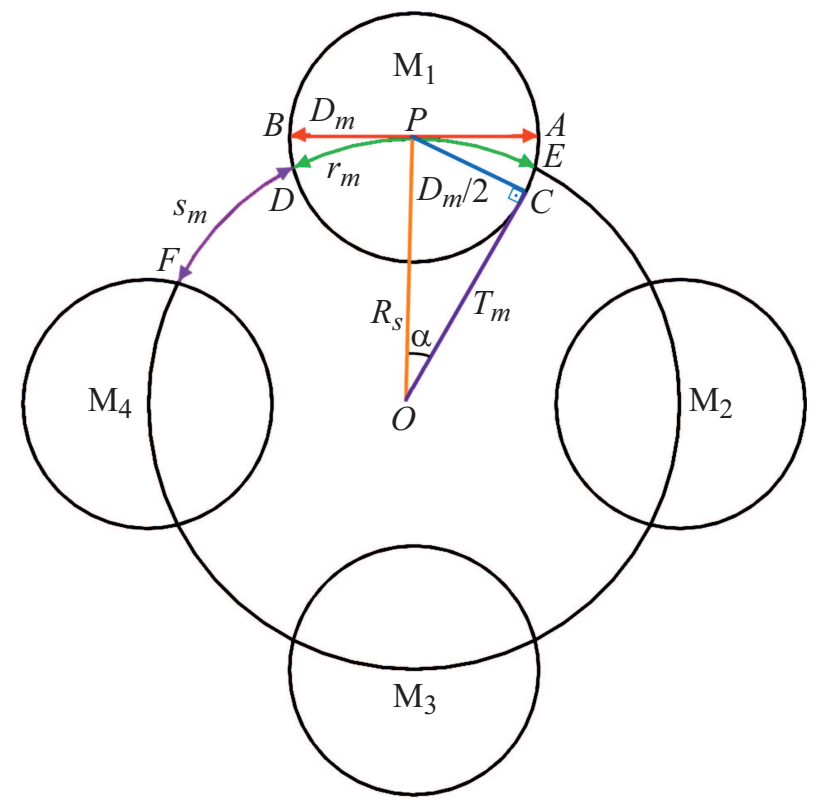

Pис. 1. Двумерное изображение четырехмагнитной сборки Хальбаха. Радиус сборки обозначен как $R_{s}$. $D_{m}$ - диаметры постоянных магнитов $\left(\mathrm{M}_{1}, \mathrm{M}_{2}, \mathrm{M}_{3}\right.$ и $\left.\mathrm{M}_{4}\right), \alpha$ - угол между касательной к магниту $\left(T_{m}\right)$ и радиусом сборки, $r_{m}$ - длина дуги, перекрытой магнитом, $s_{m}$ - длина дуги между магнитами.

при любом значении $F F$ внешний касательный угол каждого магнита можно определить как

$$
\alpha=\frac{\pi \cdot F F}{n} .
$$

В такой модели легко учитываются изменения радиусов как магнитов, так и всей сборки. Таким образом, выражение для $F F$ принимает следующий вид:

$$
F F=\frac{n}{\pi} \arcsin \left(\frac{D_{m}}{2 R_{s}}\right) .
$$

Благодаря точному математическому представлению коэффициента $F F$ проблема перекрытия соседних магнитов устраняется. В отличие от модели, принятой в литературе, данная модель не включает в себя периметр корпуса сборки (т.е. $2 \pi R_{s}$ ). В результате проблема совмешения магнитов при $F F=1$ решается так, как показано на рис. 2.

В данном исследовании проведен расчет плотности магнитного потока в центре системы на примере цилиндрических сборок Хальбаха с четырьмя и восемью магнитами, поскольку именно такая конфигурация используется чаще других, особенно в случае задачи магнитопорошковой визуализации. Для систем Хальбаха, построенных на основе уравнения (3) и выражения, приведенного в литературе [4], численный расчет плотности магнитного потока был выполнен с помощью программы COMSOL Multiphysics 5.3a (COMSOL AB, Стокгольм, Швеция).
При этом значения радиуса сборки Хальбаха $(12.5 \mathrm{~cm})$, длины постоянного магнита $(l=50 \mathrm{~cm})$ и остаточной магнитной индукции (т.е. коэффициента $B_{r}$ магнитов, равного $1.2 \mathrm{~T}$ ) поддерживались на постоянном уровне. Для оценки влияния коэффициента $F F$ на плотность магнитного потока была проведена параметрическая оптимизация. На рис. 3 представлены результаты сравнения расчетов по новой модели с результатами использования уравнения, приведенного в литературе, причем сравнение проводилось в центральных точках геометрических систем (рис. 3). Результаты, полученные с помощью новой модели, со всей очевидностью превосходят те, которые дает уравнение, приведенное в литературе. При моделировании были заданы четыре разных значения коэффициента заполнения $(0.68,0.78$, 0.88 и 1). По окончании вычислений было проведено сравнение результатов обеих моделей.

В практических приложениях фактор заполнения играет важную роль в определении зазора между магнитами, необходимого для фиксации полюсов магнитов Хальбаха в определенных точках. Неадекватность приведенного в литературе выражения становится особенно заметной в случае систем с небольшим числом магнитов, например систем Хальбаха с четырьмя и восемью магнитами, когда погрешность составляет $\sim 17.6 \%$. Однако в конфигурациях, число магнитов в которых равно или превышает 12, приведенная в литературе величина погрешности варьируется в зависимости от коэффициента заполнения в пределах $0.5-1.5 \%$. Таким образом, различие между результатами, предоставляемыми модифицированной моделью (уравнение (3)) и старой моделью (уравнение, приведенное в литературе), значительно уменьшается при использовании большого (более 10) количества магнитов.
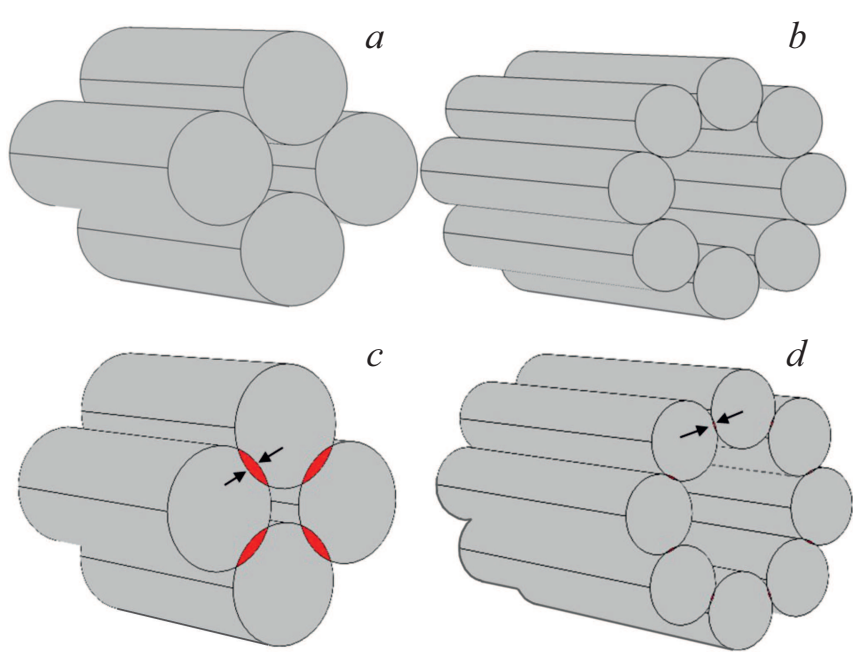

Рис. 2. Сравнительное изображение четырехмагнитной и восьмимагнитной сборок Хальбаха, рассчитанных по нашей модели (уравнение (3)) $(a, b)$ и модели, приведенной в литературе $(c, d)$, для случая $100 \%$ заполнения. Очевидно наличие перекрытия магнитов в случаях, представленных на частях $c$ и $d$. 

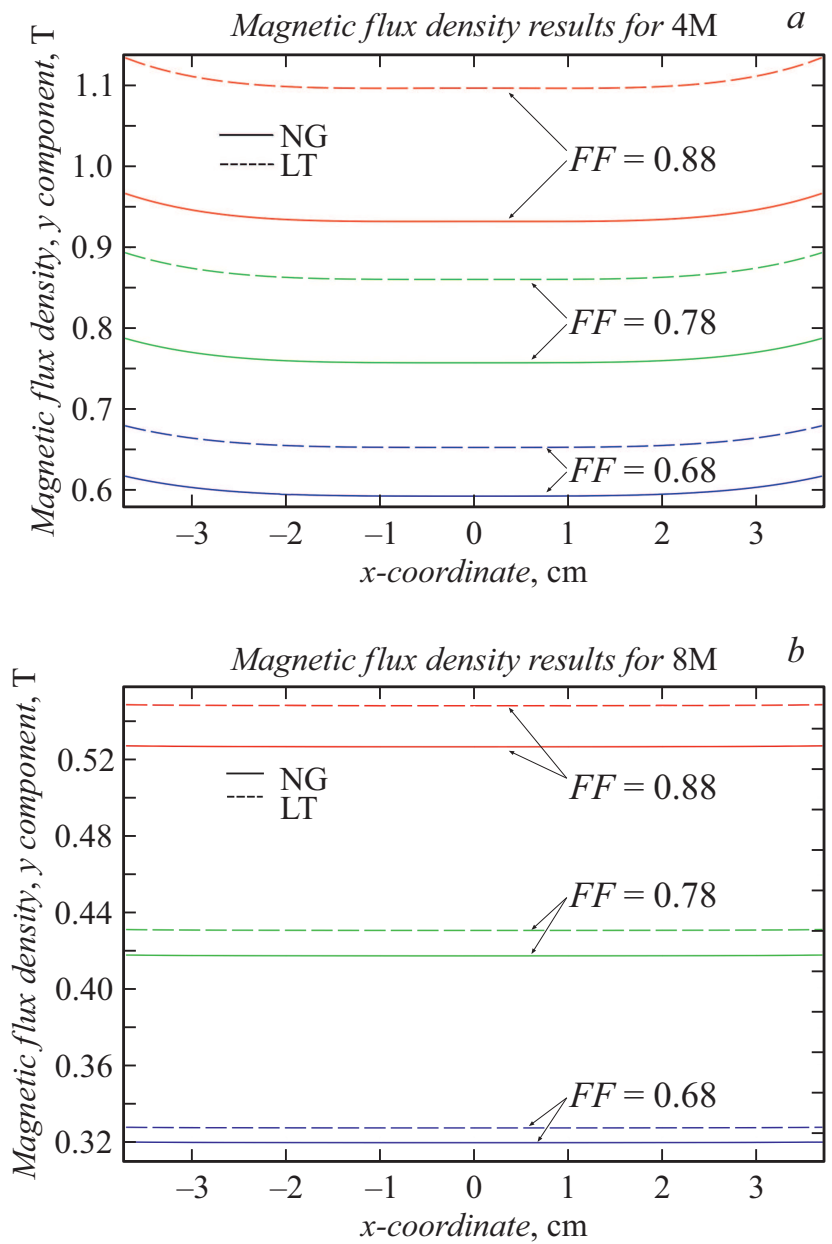

Рис. 3. Влияние значения фактора заполнения на геометрические различия между системами, смоделированными с использованием уравнения, приведенного в литературе, и нашего нового уравнения, для четырехмагнитной (4M) (a) и восьмимагнитной $(8 \mathrm{M})(b)$ сборки. $\mathrm{NG}-$ новая геометрия (сплошные линии), LT - литературные данные (штриховые линии).

Из рис. 3 видно, что различие между значениями плотности магнитного потока, полученными с помощью указанных двух уравнений при значениях коэффициента заполнения $0.68,0.78$ и 0.88 , составляет соответственно $10.08,13.53$ и $17.61 \%$ для систем с четырьмя магнитами и 2.41, 3.18 и 4.08\% для систем с восемью магнитами. Кроме того, по оптимизированному уравнению были проведены расчеты для случая $F F=1$. При этом наибольшие значения плотности магнитного потока 1.154 и $0.674 \mathrm{~T}$ были получены соответственно при четыpex и восьми магнитах (рис. 4). Центральные области сборок Хальбаха (с четырьмя и восемью магнитами) обладают высокой степенью однородности, что имеет большое значение в таких приложениях, как MRI и MPI. Однако с помощью уравнения, приведенного в литературе, спроектировать сборку Хальбаха с наибольшим (равным 1) коэффициентом $F F$ не представляется возможным.
В настоящей работе было обнаружено, что недостаток (погрешность) приведенного в литературе уравнения порождает погрешность порядка 17\% для четырехмагнитной системы Хальбаха и 4\% для восьмимагнитной. Другими словами, было показано, что для четырехмагнитной системы Хальбаха предложенная здесь численная модель обеспечивает примерно на $17 \%$ лучшую точность определения плотности магнитного потока. Разработанная нами новая формула (уравнение (3)) позволяет избавиться от перекрытия соседних магнитов в случае максимального коэффициента заполнения $(F F=1)$ и в случае наибольшей плотности магнитного потока. Таким
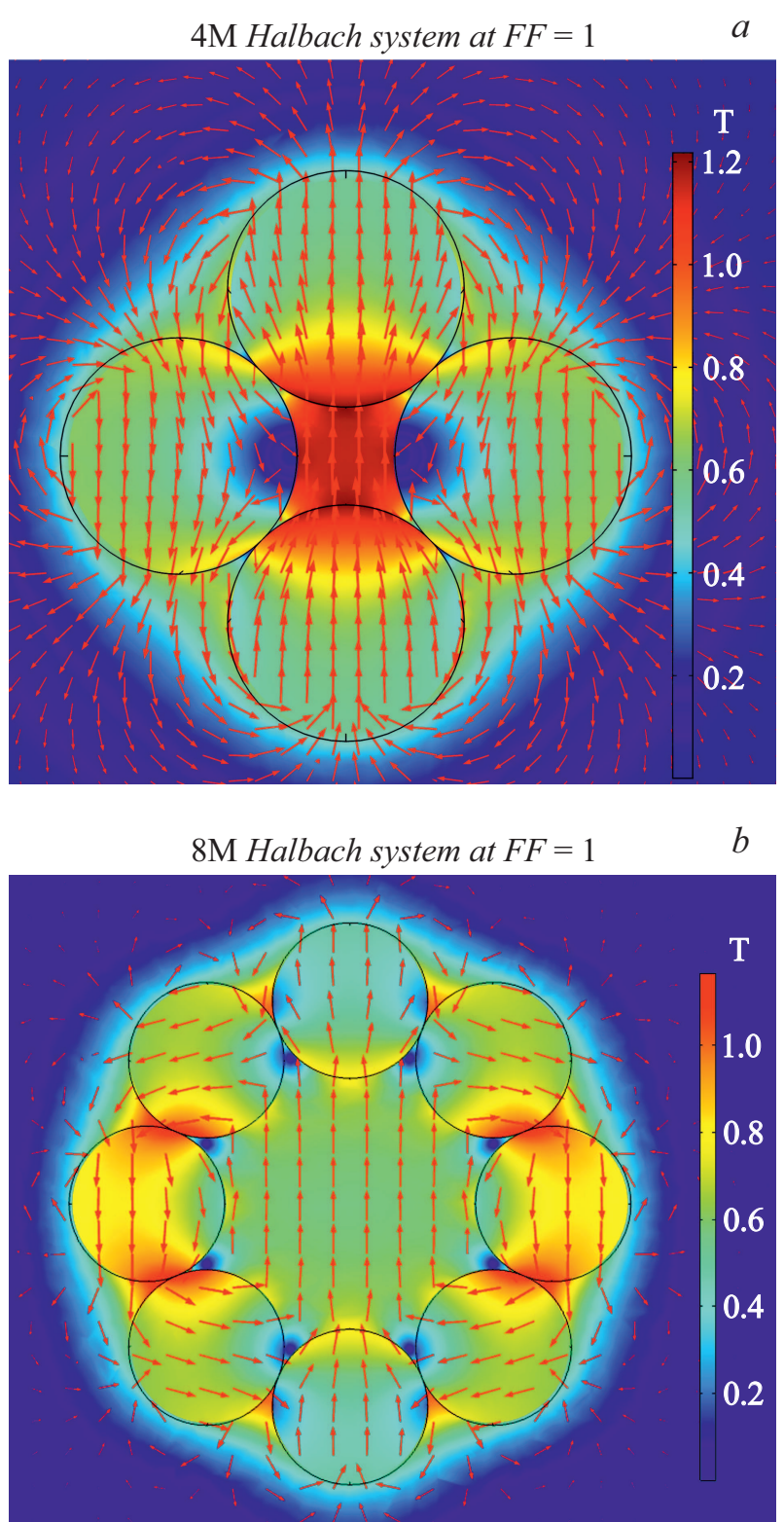

Рис. 4. Двумерные изображения плотности магнитных потоков для четырехмагнитной $(4 \mathrm{M})$ и восьмимагнитной $(8 \mathrm{M})$ геометрии для приложений с использованием однородного магнитного поля при $100 \%$ заполнении. $a-$ значение $1.154 \mathrm{~T}$ получено в центре четырехмагнитной сборки, $b-$ значение 0.674 T получено в центре восьмимагнитной сборки. 
образом, результаты данного исследования позволяют устранить погрешность и другие недостатки модели, задаваемой приведенным в литературе уравнением.

\section{Финансирование работы}

Финансовая поддержка работы осуществлялась Советом по научно-техническим исследованиям Турции (Scientific and Technological Research Council of Turkey) (грант TUBITAK 115E776\&115E777).

\section{Конфликт интересов}

Авторы заявляют, что у них нет конфликта интересов.

\section{Список литературы}

[1] J.C. Mallinson, IEEE Trans. Magn., 9 (4), 678 (1973).

[2] K. Halbach, IEEE Trans. Nucl. Sci., 26 (3), 3882 (1979).

[3] K. Halbach, Nucl. Instrum. Meth., 169 (1), 1 (1980).

[4] M.W. Vogel, A. Giorni, V. Vegh, R. Pellicer-Guridi, D.C. Reutens, PLoS ONE, 11 (6), e0157040 (2016).

[5] K. Turek, P. Liszkowski, J. Magn. Res., 238, 52 (2014).

[6] C.W. Windt, H. Soltner, D. van Dusschoten, P. Blümler, J. Magn. Res., 208 (1), 27 (2011).

[7] S. Anferova, V. Anferov, J. Arnold, E. Talnishnikh, M.A. Voda, K. Kupferschläger, B. Blümich, Magn. Res. Imaging, 25 (4), 474 (2007).

[8] C.Z. Cooley, J.P. Stockmann, B.D. Armstrong, M. Sarracanie, M.H. Lev, M.S. Rosen, L.L. Wald, Magn. Res. Med., 73 (2), 872 (2015).

[9] C.Z. Cooley, M.W. Haskell, S.F. Cauley, C. Sappo, C.D. Lapierre, C.G. Ha, L.L. Wald, IEEE Trans. Magn., 54 (1), 5100112 (2018).

[10] J. Konkle, P. Goodwill, S. Conolly, in Medical imaging 2011: biomedical applications in molecular, structural, and functional imaging, Proc. SPIE, 7965, 79650X (2011).

[11] M. Weber, J. Beuke, A. von Gladiss, K. Gräfe, P. Vogel, V.C. Behr, T.M. Buzug, Int. J. Magn. Part. Imaging, 4 (2), 1811004 (2018).

[12] H. Bagheri, C.A. Kierans, K.J. Nelson, B.A. Andrade, C.L. Wong, A.L. Frederick, M.E. Hayden, in 5th Int. Workshop on magnetic particle imaging (IWMPI) (IEEE, 2015), p. 1-1.

[13] P. Babinec, A. Krafčík, M. Babincová, J. Rosenecker, Med. Biol. Eng. Comput., 48 (8), 745 (2010).

[14] A. Sarwar, A. Nemirovski, B. Shapiro, J. Magn. Magn. Mater., 324 (5), 742 (2012).

[15] H. Soltner, P. Blümler, Concepts Magn. Res. A, 36A (4), 211 (2010).

[16] F. Balc1, Master thesis (Yıldiz Technical University, Istanbul, Turkey, 2020). 